\title{
Study of Phototoxicity of LED Light for Colon Cancer
}

\author{
Yu Wen Hung ${ }^{1,2}$, Cheng Sheng Tsung ${ }^{3}$, Ching Feng Chiu ${ }^{4}$, Chien Chao Chiu ${ }^{5}$, Hsuan Wen Chiu ${ }^{6}$, Yu Hsing Lin ${ }^{2}$, Wei \\ Huang Tsai ${ }^{7}$ and Shao Wen Hung ${ }^{* 2,5}$
}

${ }^{1}$ Institute of Cellular and System Medicine, National Health Research Institutes, Maioli 350, Taiwan

${ }^{2}$ Nursing Department of Yuanpei University, Xiangshan, Hsinchu 300, Taiwan

${ }^{3}$ Department of Materials Science and Engineering, National Chung Hsing University, Taichung 402, Taiwan

${ }^{4}$ Graduate Institute of Metabolism and Obesity Sciences, College of Nutrition, Taipei Medical University, Taipei 110, Taiwan

${ }^{5}$ Division of Animal Industry, Animal Technology Laboratories, Agricultural Technology Research Institute, Xiangshan, Hsinchu 300, Taiwan

${ }^{6}$ Department of Biotechnology and Bioindustry Sciences, College of Bioscience and Biotechnology, National Cheng Kung University, Tainan 701, Taiwan

${ }^{7}$ Department of Science and Technology, Council of Agriculture, Executive Yuan, Taipei 100, Taiwan

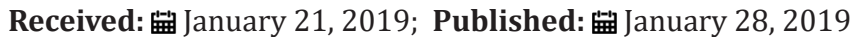

*Corresponding author: Shao Wen Hung, Division of Animal Industry, Animal Technology Laboratories, Agricultural Technology Research Institute, Xiangshan, Taiwan

\begin{abstract}
Colon cancer is very important cancer worldwide. Although many therapies and therapeutic strategies are applied in the patients with cancer however, the deaths of patients with cancer maintain in the top of the dead people worldwide. The visible light at a wavelength corresponding to the drug absorption activates these agents and induces highly cytotoxic products was verified. Application of the visible light at a wavelength is a potential for cancer therapy. In this study, in vitro and in vivo data were demonstrated that LED light at $450 \mathrm{~nm}$ induces highly cytotoxic ability and suppresses the growth of colon cancer in the CT- 26 bearing mice. Taken together, LED light at $450 \mathrm{~nm}$ has the potential to be developed as a new therapeutic method for colon cancer in the future.
\end{abstract}

Keywords: Colon cancer; In vitro; In vivo; LED light; Phototoxicity

\section{Mini Review}

\section{Phototoxicity on Colon Cancer}

Cancer may be viewed as a disease that progresses through multiple stages that involve initiation, promotion, progression, and metastasis [1]. Colon cancer is very important cancer worldwide. Although many therapies and therapeutic strategies are applied in the patients with cancer, however, the deaths of patients with cancer maintain in the top of the dead people worldwide [2]. Previous researchers have demonstrated that photodynamic therapy using cancer designed photosensitizers and laser beams has been increasingly used in clinical medicine. The visible light at a wavelength corresponding to the drug absorption activates these agents and induces highly cytotoxic products: singlet molecular oxygen and oxygen radicals. The LED light induced cell damage was wavelength dependent, but not energy dependent [3]. Therefore, novel therapies and therapeutic strategies are needed for the cancer therapy in the future. The objective of this investigation was to $R \& D$ of the novel therapies and therapeutic strategies against colon cancer via this novel therapy. In addition, we will wish to apply this phototoxic therapeutic method against the tumor growth in the deep body cavities in the future. Finally, we hope this novel therapies also can be applied to suppress tumor growth, metastasis, and recrudescence.

\section{Effect of LED Light at $450 \mathrm{~nm}$ Wavelength with on Colon Cancer Growth In vitro and In vivo}

Human and murine colon carcinoma cell lines used were HT-29 (ATCC ${ }^{\circledR}$ number: HTB-38 ${ }^{\mathrm{TM}}$ ) and CT-26 (ATCC $^{\circledR}$ number: CRL-2638 ${ }^{\text {TM }}$ ). HT-29 and CT-26 were respectively cultured in McCoy's 5a medium modified and RPMI-1640 medium (GIBCO ${ }^{\circledR}$ ) and were maintained in its specific media supplemented with $10 \%$ fetal bovine serum (FBS), $100 \mathrm{U} / \mathrm{ml}$ penicillin and $100 \mathrm{mg} /$ $\mathrm{ml}$ streptomycin (Invitrogen), in a humidified $5 \% \mathrm{CO}_{2}$ incubator at $37^{\circ} \mathrm{C}$. Effect of phototoxicity under LED lighting for human and murine colon cancer cell lines in vitro was evaluated. Cell morphology was observed between the in vitro experiments. Cells 
were treated with LED lighting $8 \mathrm{hr}$ per day for 14 days in the 6 well culture plate. Observation of cell morphology with/without LED lighting $(11,700 \mathrm{~lm}$; Figure 1A) was by light microscope (Axiovert $200 \mathrm{M}$, Zeiss). Data were shown that LED light significantly induce cell death of HT-29 and CT-26 cells after 4 hours LED light exposure. Between 4 to 12 hours LED light exposure, all HT-29 and CT-26 cells were died according to their cell morphology (Figure 1B).

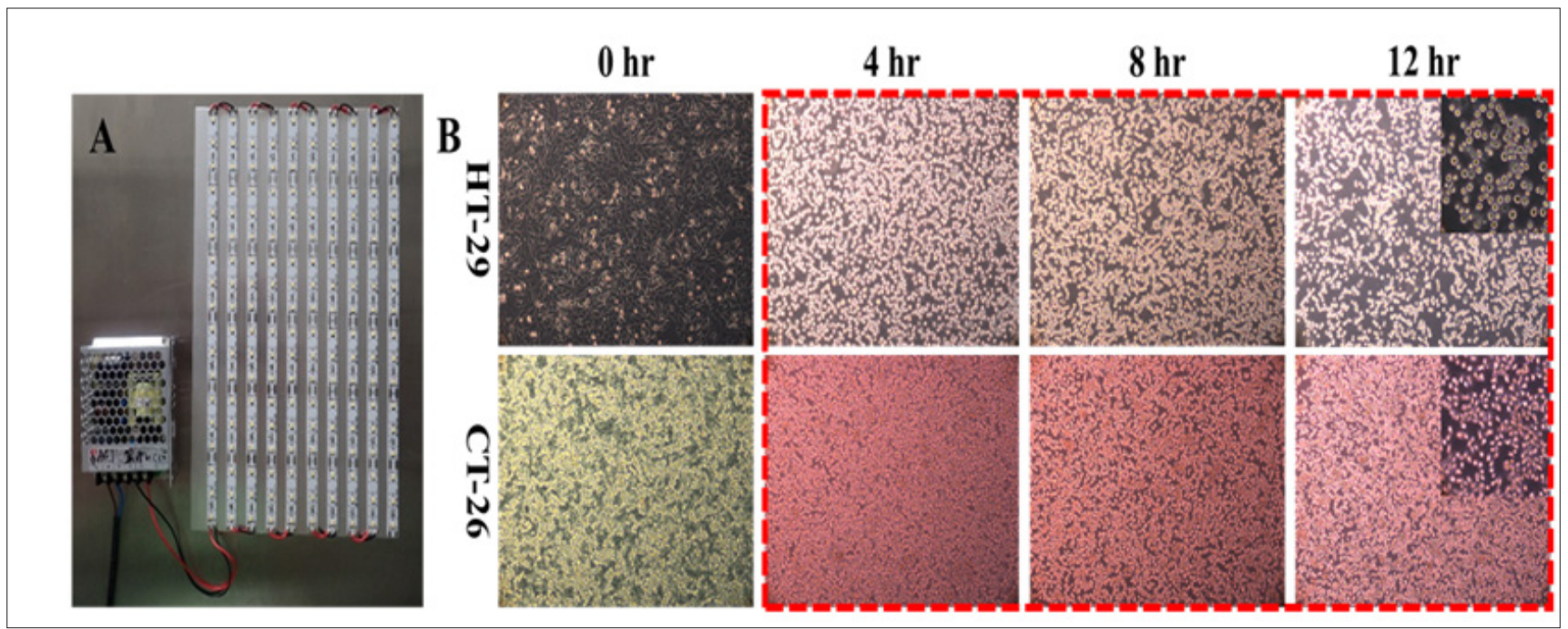

Figure 1: Phototoxic device and in vitro effects. (A) Appearance of LED light. (B) LED light is phototoxic for colon cancer cell lines, HT-29 and CT-26, by direct cytocidal effect in vitro. HT-29: Human colon cancer cell line; CT-26: Murine colon cancer cell line.

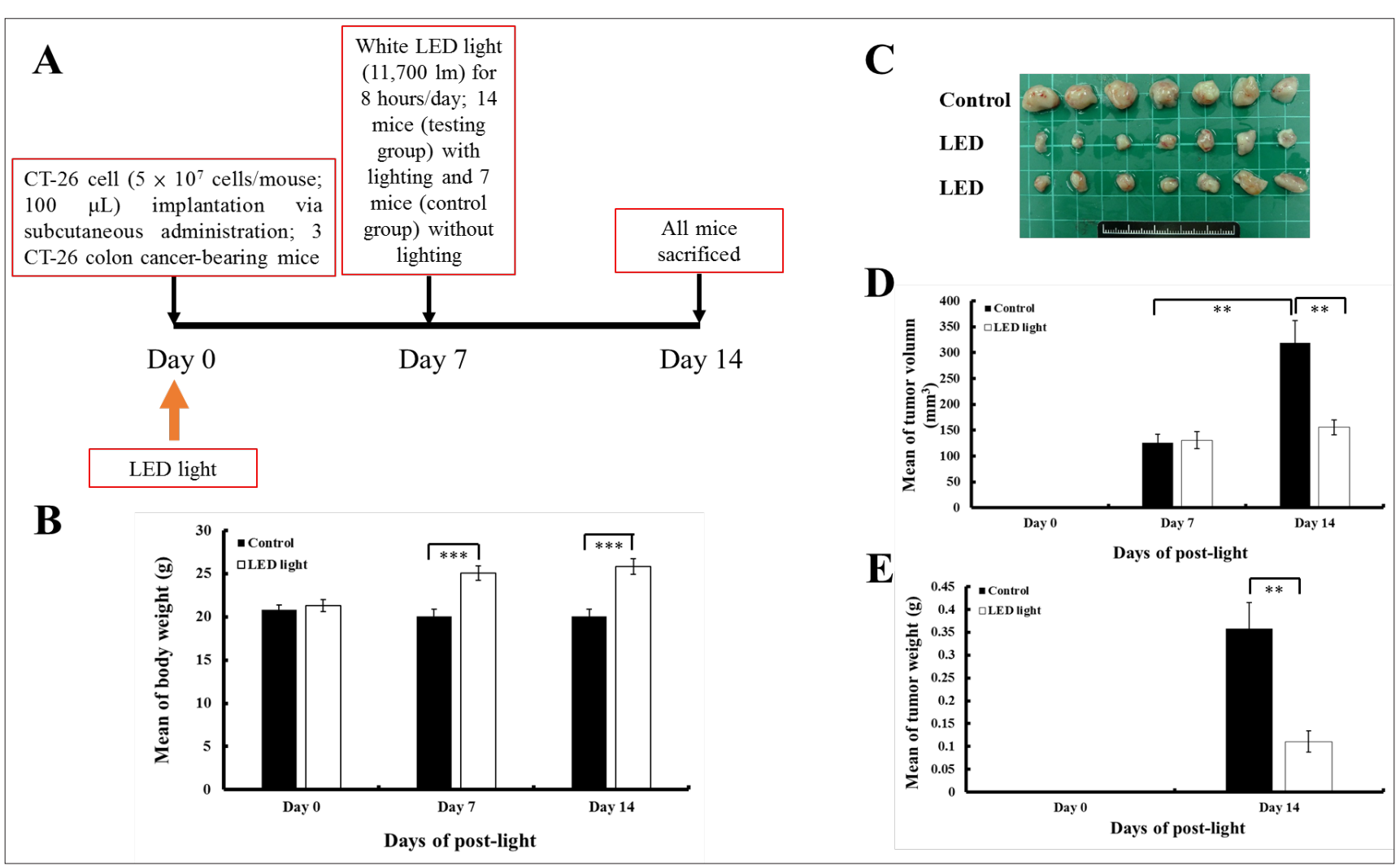

Figure 2: Design of this study and phototoxic device and in vivo effects. (A) Design of this study. (B) LED light is benefit for the increase of body weight of mice. ${ }^{* * *} p<0.001$. (C) Gross appearance of CT-26 colon tumor mass. (D) LED light is photo suppression for the tumor volume of CT-26 murine colon carcinoma in an ectopic allograft murine model in vivo. ${ }^{* *} p<0.01$. (E) LED light is photo suppression for the tumor weight of CT-26 murine colon carcinoma in an ectopic allograft murine model in vivo. ${ }^{* *} p<0.01$. No. of mice in LED group is 14; No. of mice in Control group is 7 . 
Animal care and all in vivo experiments were performed in compliance with the guidelines of the Agricultural Technology Research Institute (ATRI): Institutional Animal Care \& Utilization Committee. BALB/c mice (6 to 8 weeks old) were purchased from National Laboratory Animal Center, Taipei, Taiwan. Mice were provided a standard laboratory diet and distilled water and kept on a $12 \mathrm{~h}$ light/dark cycle at $25 \pm 2^{\circ} \mathrm{C}$ in the GLP Animal Test Room, Animal Technology Laboratories, ATRI, Miaoli, Taiwan. The experimental design in vivo was performed. To test the effects of LED light on CT-26 cells in vivo, CT-26 was established by subcutaneous implantation of CT-26 cells into BALB/c mice. BALB/c mice ( $\mathrm{n}=7$ mice/control group; 14 mice/LED group) were randomly assigned to two groups: (1) a control group, vehicle with CT-26 cell implantation and without LED lighting; (2) a LED groups, CT-26 cell implantation with LED lighting ( $8 \mathrm{hr} /$ day for 2 weeks). After 7 days CT-26 cell implantation, LED lighting was performed (Figure 2A). All animal experiments were approved by the Institutional Animal Care and Use Committee of ATRI, Hsinchu, Taiwan (approval No.: 107025). CT-26 cells $\left(5 \times 10^{6}\right.$ cells per mouse) were suspended in $100 \mathrm{ml}$ of serum free RPMI-1640 medium and injected subcutaneously into mice, aged 6 to 8 weeks. When tumors were detected, tumor volume was measured using the formula: $1 / 2 \times$ the largest diameter $\times$ (the smallest diameter) ${ }^{2}$, as reported in previous cancer studies [4,5]. Measurement of tumor volume was periodically performed with calipers. At the end of the experiment, all mice in each group were sacrificed and tumor mass were collected. Herein, LED light with wavelength at 450 $\mathrm{nm}$ significantly elevated body weight of mice (Figure $2 \mathrm{~B}$ ). In vivo data were demonstrated that LED light with wavelength at $450 \mathrm{~nm}$ significantly suppressed CT-26 tumor weight and tumor volume in BALB/c mice with an ectopic allograft model (Figure 2C-2E).

\section{References}

1. Jemal A, Siegel R, Xu J, Ward E (2010) Cancer statistics, 2010. CA Cancer J Clin 60(5): 277-300.

2. Siegel RL, Kimberly DM, Ahmedin J (2017) Cancer Statistics, 2017. CA Cancer J Clin 67(1): 7-30.

3. Sparsa A, Faucher K, Sol V, Durox H, Boulinguez S, et al. (2010) Blue light is phototoxic for B16F10 murine melanoma and bovine endothelial cell lines by direct cytocidal effect. Anticancer Res 30(1): 143-148.

4. Chen TA, Wang JL, Hung SW, Chu CL, Cheng YC, et al. (2011) Recombinant VP1, an Akt inhibitor, suppresses progression of hepatocellular carcinoma by inducing apoptosis and modulation of CCL2 production. PLoS One 6: e23317.

5. Hung SW, Chiu CF, Chen TA, Chu CL, Huang CC, et al. (2012) Recombinant viral protein vp1 suppresses her-2 expression and migration/metastasis of breast cancer. Breast Cancer Res Treat 136(1): 89-105.
ISSN: 2574-1241

DOI: 10.26717/BJSTR.2019.13.002443

Shao Wen Hung. Biomed J Sci \& Tech Res

This work is licensed under Creative Commons Attribution 4.0 License

Submission Link: https://biomedres.us/submit-manuscript.php

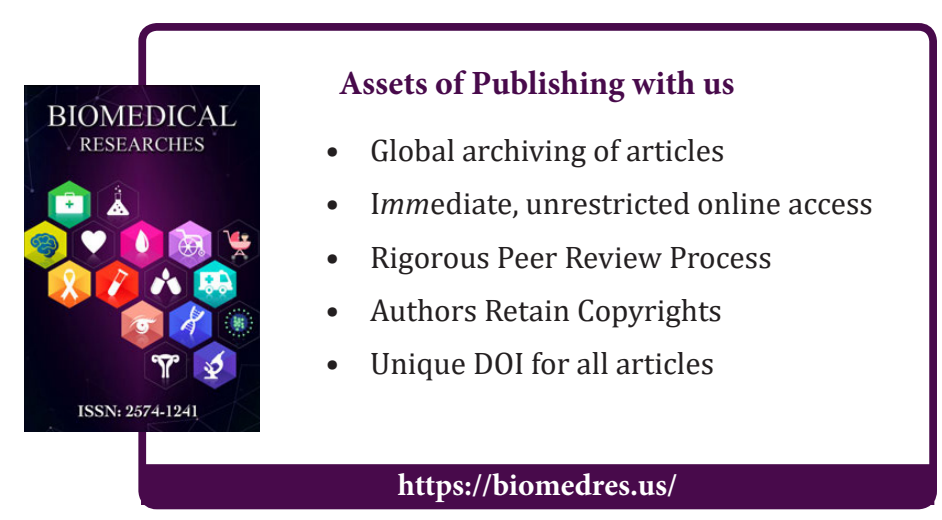

